\title{
Reproducción, muda y desarrollo de la langosta de Juan Fernández, Jasus frontalis, en estanques de cultivo
}

\author{
Enrique Dupré $\mathrm{M}$. \\ Departamento de Biología Marina \\ Facultad de Ciencias del Mar, Universidad Católica del Norte \\ Casilla 117, Coquimbo, Chile \\ E-mail: edupre@socompa.cecun.ucn.cl
}

Recibido: 23 marzo 1999; versión corregida: 10 mayo 1999; aceptado: 5 noviembre 1999

\begin{abstract}
RESUMEN: Se determinó el período de muda, apareamiento, portación de huevos, eclosión de phyllosomas y duración del desarrollo larval, de Jasus frontalis, a diferentes temperaturas. Todas las determinaciones se realizaron en individuos sexualmente maduros y en estanques de 2.0001 para los períodos de muda y en estanques de 501 para la eclosión de larvas y desarrollo larval. Este último también se realizó en vasos de vidrio de 11 a diferentes temperaturas. Se determinó que las mudas de hembras y machos no ocurren en el mismo período. Los machos mudan entre febrero y marzo de cada año cuando la temperatura alcanza los $17,0 \pm 0,5^{\circ} \mathrm{C}$ y $16,9 \pm 0,4^{\circ} \mathrm{C}$ respectivamente en cambio las hembras mudan desde la mitad de abril hasta mediados de junio con temperaturas que varían entre $15,0 \pm 0,5^{\circ} \mathrm{C}$ y 13,1 $\pm 0,2^{\circ} \mathrm{C}$ respectivamente. El crecimiento de la longitud cefalotorácica, tanto en machos como hembras fue de 1,3 a 3,6 mm por muda y el endurecimiento del caparazón ocurre dentro de los primeros tres días.

El inicio del período de portación de huevos ocurre entre junio y agosto, y la eclosión larval entre octubre y diciembre de cada año. El desarrollo embrionario tiene una duración de $115 \pm 3$ y $78 \pm 9$ días a $13^{\circ} \mathrm{C}$ y $18^{\circ} \mathrm{C}$ respectivamente. En todos los casos observados, la eclosión ocurrió después del atardecer y antes de medianoche. La hembra participa activamente en la liberación de las larvas naupliosomas, las cuales después de 10-20 min. de activa natación mudan y originan la primera phyllosoma.

Después de 5 días de iniciada la eclosión se observó que entre 2 y $8 \%$ de la masa total de huevos portados permanecen adheridos a los pleópodos junto a gran cantidad de cápsulas vacías. La totalidad de estos huevos remanentes se encuentran en el primer estado de desarrollo. Los seis primeros estados larvarios obtenidos demoraron entre 56 y 92 días dependiendo de la temperatura del cultivo. A $14-15^{\circ} \mathrm{C}$ el $5^{\circ}$ estado se obtuvo a los 77 días. El sexto estado se obtuvo a los 79 días a $17-18^{\circ} \mathrm{C}$, a $19-20^{\circ} \mathrm{C} 69$ días y a $20-21^{\circ} \mathrm{C} 56$ días.
\end{abstract}

Palabras claves: ciclo reproductivo, muda, eclosión, desarrollo larval, Jasus frontalis.

\section{Reproduction, molt and development of the spiny lobster of Juan Fernandez, Jasus frontalis, in laboratory conditions}

\footnotetext{
ABSIRACT: In the present study the molting period, mating, egg carrying, phyllosome eclosion and duration of larval development of the spiny lobster Jasus frontalis were determined at different temperatures. All the observations were carried out using sexually mature individuals in 20001 (molting period), and 501 ( larval eclosion and development) seawater tanks. Larval development was also observed in vitro in one-liter glass beakers.

It was found that males and females did not molt within the same time periods. Males molted between February and March every year when the temperature reached $17.0 \pm 0.5^{\circ} \mathrm{C}$ or $16.9 \pm 0.4^{\circ} \mathrm{C}$. Females molted from the middle of April to the middle of June when temperatures varied between $15 \pm 0.5^{\circ} \mathrm{C}$ and $13.1 \pm 0.2^{\circ} \mathrm{C}$. Growth at each molt was 1.3 to $3.6 \mathrm{~mm}$ in both males and females, and hardening of the new carapace occurred within three days.

Initiation of the egg carrying (berried) stage occurred between June and August, and eclosion of larvae between October and December of each year. Embryonic development lasted $115 \pm 3$ days at $13^{\circ} \mathrm{C}$ and $78 \pm 9$ days at $18^{\circ} \mathrm{C}$. In all cases observed, eclosion occurred after sunset and prior to midnight. The female participates actively in liberation of the naupliosome larvae, which swim actively for 10-20 minutes, after which they molt and become the first phyllosome larvae.
}

Key words: reproductive cycle, molt, hatching, larval development, Jasus frontalis. 


\section{INTRODUCCIÓN}

Los primeros estudios realizados sobre la langosta de Juan Fernández, Jasus frontalis, a partir de 1980, se refieren principalmente a parámetros biológicopesqueros, como crecimiento (Arana y Martínez, 1985; Arana y Olate, 2000), mortalidad y rendimiento (Díaz y Arana, 1985), captura (Arana y Melo, 1973; Larraín y Yáñez, 1985; Arana y Vega, 2000; Yáñez et al., 1985; Yañez et al., 2000), dinámica y estructura poblacional (Gaete y Arana, 1985; Arana y Olate, 2000). En cambio, los estudios relativos a su reproducción y desarrollo son escasos. Arana et al. (1982, 1985) determinaron la talla de primera madurez sexual, la fecundidad y describieron diez estados de desarrollo embrionario sobre la base de las características morfológicas y grado de coloración de los embriones. La descripción de algunos estados larvales (VII, XI, XIIIA y XIIIB) fue realizada a partir de larvas obtenidas en muestras planctónicas colectadas en las cercanías de la isla Robinson Crusoe (Báez, 1973).

Estudios recientes han permitido avanzar en el conocimiento de la biología de la reproducción y desarrollo de esta especie. Se caracterizó cada uno de los estados del desarrollo embrionario tanto a microscopía óptica (Dupré, 1988) como electrónica de barrido (Tavonatti, 1998); se evaluó la maduración ovárica inducida por hormonas exógenas (Elorza, 1998); se estableció la organización estructural del ovario (Elorza y Dupré, 2000) y se determinó la duración y características de los diferentes estados del ciclo de muda mediante el análisis de los pleópodos (Elorza y Dupré, 1996). Con relación al desarrollo larval, se analizó la sobrevivencia de los tres primeros estados de phyllosoma bajo diferentes tipos de alimentación; se describió el primer estado de phyllosoma (Dupré, 1996) y en cultivos experimentales mantenidos durante tres meses se logró caracterizar en forma general los primeros cinco estados de phyllosoma (Dupré y Guisado, 1996), detectándose una alta mortalidad debido a la alta contaminación de las larvas con vibrios y hongos filamentosos (Dupré, datos no publicados).

Aun cuando estos avances constituyen valiosos aportes al conocimiento de la biología de esta especie, aún no es posible establecer su ciclo reproductivo completo. Por esto el presente estudio realiza una secuencia cronológica de eventos que ocurren durante el ciclo anual de ejemplares mantenidos en cautiverio, señalando el período en que ocurren las mudas en machos y hembras, el período de apareamiento, el tiempo transcurrido entre el apareamiento e inicio de la portación de huevos, el períodos de eclosión y el tiempo que demoran el desarrollo embrionario y larval a diferentes temperaturas. Todos estos objetivos contribuyen a tener un conocimiento aproximado de lo que ocurre con el ciclo reproductivo de esta especie en la naturaleza.

Otros aspectos importantes sobre la reproducción, como el cortejo de apareamiento, comportamiento reproductivo, período del año en que ocurre el apareamiento en la naturaleza, momento en que ocurre el apareamiento después de la muda y aspectos sobre su desarrollo, como la presencia y distribución de larvas phyllosomas en el plancton alrededor del archipiélago (Báez, 1973; Palma et al., 1976; Palma, 1985), deberán ser determinados en estudio posteriores.

\section{MATERIALES Y MÉTODOS}

Entre 1994 y 1995 se capturaron ejemplares maduros de langosta de Juan Fernández (Jasus frontalis) alrededor de la isla Robinson Crusoe, entre 100 y $150 \mathrm{~m}$ de profundidad, los cuales fueron transportados vivos al laboratorio de cultivos de la Universidad Católica del Norte, Coquimbo. Los experimentos realizados se desarrollaron entre 1995 y 1996.

Los ejemplares, 20 hembras y 30 machos, cuyas longitudes cefalotorácicas estuvieron comprendidas entre 85,5 y $99,2 \mathrm{~mm}$ en hembras y 102,6 y 116,7 $\mathrm{mm}$ en machos, fueron distribuidos en cuatro estanques circulares de 5001 y mantenidos con circulación de agua de mar filtrada (50 mm) (AMF) en circuito abierto, a temperatura ambiente y aireación constante. Cada dos días fueron alimentados con trozos de pescado congelado y al día siguiente fueron extraídos los residuos del fondo. Los estanques 1 a 3 contenían 7, 9 y 9 hembras y 7, 5 y 3 machos, respectivamente. El cuarto estanque contenía $1 \mathrm{ma}-$ cho sexualmente maduro y 6 hembras, las cuales fueron transferidas de sus propios estanque después de 3 días de ocurrida la muda.

Los estanques 1 a 3 fueron revisados diariamente a fin de extraer las mudas producidas y los individuos recientemente mudados fueron transferidos a un estanque de 501 hasta el endurecimiento del caparazón. El estanque 4 se revisó cada 4 hr durante el día, para detectar apareamientos o presencia de huevos en los pleópodos. 
Se registró cada muda, presencia de huevos en los pleópodos y tiempo de endurecimiento del caparazón. El aumento de tamaño de los individuos recientemente mudados fue determinado midiendo el caparazón, con un pie de metro, desde el margen post-orbital al extremo distal del cefalotórax.

La temperatura del agua de los estanques varió entre $13,1^{\circ} \mathrm{C}$ en junio de 1995 y $17,8^{\circ} \mathrm{C}$ en diciembre de 1996. El desarrollo embrionario y larval se realizó en cultivos a diferentes temperaturas $(13,16$ y $\left.18^{\circ} \mathrm{C}\right)$. La determinación de los estados del desarrollo embrionario se registró según Dupré (1988), para lo cual se observó y midió bajo microscopio con 40 aumentos grupos de 10 a 15 embriones provenientes de 6 hembras ovíferas. Para determinar el tiempo que dura la liberación de larvas se ubicó a cada hembra que presentaba sus embriones en el estado final del desarrollo en estanques de eclosión a fin de recolectar las larvas (Dupré, 1988). Estos consisten en estanques cilíndricos de 50 litros, con flujo constante de AMF, de base plana y un tubo lateral de salida de agua superficial ubicado a 35 $\mathrm{cm}$ de la base. El flujo de salida que lleva las larvas recién liberadas, cae dentro de un tamiz de $300 \mathrm{~mm}$ semi-sumergido en agua de mar ubicado a la salida del tubo lateral.
Después de lavar con agua filtrada las larvas recuperadas en el tamiz fueron ubicadas individualmente en vasos de precipitado de $11 \mathrm{y}$ en grupos de 30-50 larvas/1 en estanques de cultivos de 501.

Diariamente se realizó el recambio total del agua, se les administró nauplius de Artemia y se registró la temperatura. Para determinar la duración de cada estado de desarrollo larval y porcentaje de sobrevivencia se registró diariamente la presencia de mudas e individuos muertos.

Los cultivos larvales se realizaron a los siguientes rangos de temperatura: $14-15^{\circ} \mathrm{C}, 17-18^{\circ} \mathrm{C}, 19$ $20^{\circ} \mathrm{C}$ y $20-21^{\circ} \mathrm{C}$, los cuales se mantuvieron mediante un termostatos Jager.

\section{RESULTADOS}

\section{Ciclo reproductivo y muda}

El ciclo reproductivo y muda anual de la langosta de Juan Fernández (J. frontalis), está representado en la Fig. 1. Se observó que las mudas, las cuales se producen antes del apareamiento, ocurren en los machos entre febrero y marzo de cada año cuando la temperatura alcanza $17,0^{\circ} \mathrm{C} \pm 0,5$ y $16,9^{\circ} \mathrm{C} \pm 0,4$

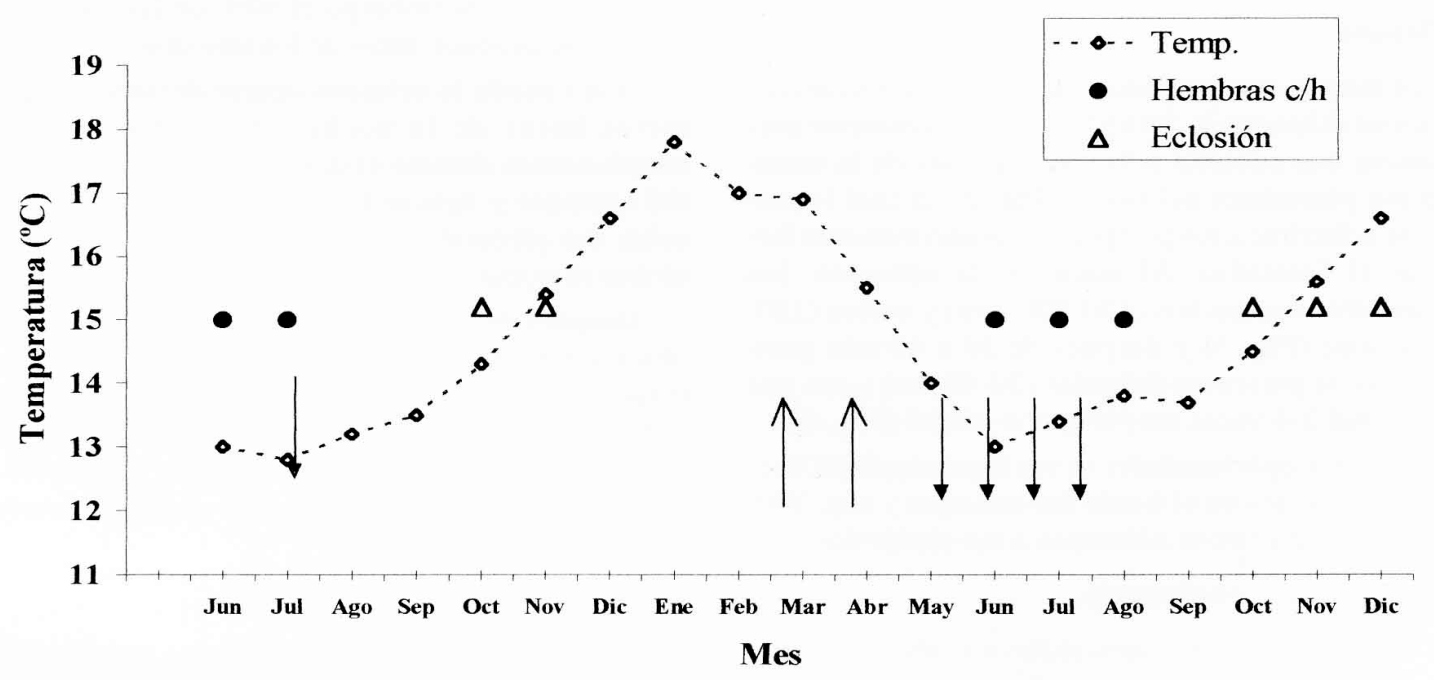

Figura 1. Ciclo reproductivo y muda anual de la langosta de Juan Fernández, Jasus frontalis en estanques de cultivo. $($ Temp. $)=$ Temperatura del agua de los estanques; $(\quad)=$ muda de machos; $(\downarrow)=$ muda de hembras; $(\bullet)$ = comienzo de portación de huevos; (D) = inicio de eclosión de larvas.

Figure 1. Reproductive and moult cycle of the spiny lobster of Juan Fernández, Jasus frontalis, reared in laboratory. $($ Temp. $)=$ Water tank temperature; ()$^{\uparrow}=$ Male moults; $(\quad)=$ female moults; $(\bullet)=$ egg berried females and $(D)=$ hatching of larvae. 
respectivamente. Las mudas de las hembras ocurrieron desde la mitad de abril hasta mediados de junio, con temperaturas que variaron entre $15,0^{\circ} \mathrm{C} \pm 0,5 \mathrm{y}$ $13,1^{\circ} \mathrm{C} \pm 0,2$ respectivamente. El crecimiento por muda varió entre 1,3 y $3,6 \mathrm{~mm}$ en los machos y entre 1,5 y $3,1 \mathrm{~mm}$ en las hembras. En promedio, los machos aumentaron en un 2,3\% de su longitud y las hembras en un $2,7 \%$. El endurecimiento del caparazón ocurre lentamente hasta el $3^{\text {er }}-5^{\circ}$ día. Durante este período, el individuo no se alimentó y su actividad disminuyó considerablemente.

\section{Apareamiento}

Aun cuando no se observó ningún apareamiento, se determinó que ocurren con mayor frecuencia durante la noche ya que no se advirtieron cópulas durante el día. En todos los casos el apareamiento se realizó entre una hembra post-mudada y un macho de mayor talla. Posiblemente el apareamiento ocurre entre 3 y 20 días post-muda, ya que en todos los casos se detectó la presencia de hembras ovígeras transcurridos 21 días de realizada la ecdisis.

El macho que se ubicó en el estanque 4, a medida que se le incorporaron las hembras, fue capaz de fecundar las 6 hembras que se introdujeron al estanque, ya que todas ellas presentaron huevos viables en sus pleópodos entre junio y fines de agosto.

\section{Desove}

Los huevos recientemente desovados son esféricos con un diámetro de 590-610 mm. Externamente presentan una cubierta adhesiva separada de la membrana plasmática del huevo (Fig. 2), la cual le permite adherirse a los pleópodos y posteriormente formar el funiculus. Al inicio de la adhesión, los funiculus son anchos (450-500 mm) y cortos (120$250 \mathrm{~mm}$ ) (Fig. 3) y después de 30 a 40 min postdesove se presentan delgados $(30-40 \mathrm{~mm})$ y con una longitud 3-4 veces mayor que el inicial (Fig. 4).

En dos oportunidades se vio la presencia de huevos u ovocitos en el fondo del estanque y sólo 100300 permanecieron adheridos a los pleópodos.

\section{Desarrollo embrionario}

El desarrollo embrionario se llevó a cabo en promedio entre 115 y 76 días post-desove a temperaturas de $13^{\circ} \mathrm{C}$ y $18^{\circ} \mathrm{C}$ respectivamente (Fig. 5). A 18,16 y $13^{\circ} \mathrm{C}$ demoraron en promedio 79,85 y 115 días, correspondientemente. Las mayores variaciones se registraron en el primer estado cuando son incubados a diferentes temperaturas $\left(29\right.$ días a $13^{\circ} \mathrm{C} ; 22$ días a $16^{\circ} \mathrm{C}$ y 19 días a $18^{\circ} \mathrm{C}$ ). Entre el estado 4 y 7 del desarrollo las variaciones atribuibles a la temperatura no son significativas cuando son incubados a 16 y $18^{\circ} \mathrm{C}$ (entre 10,5 y 10,0 días en promedio respectivamente), en cambio los estados 2 y 4 el promedio fue de 11 y 8,6 días, respectivamente.

\section{Eclosión de las larvas}

La larva que eclosiona del huevo corresponde a un naupliosoma. Después de 20 min de activa natación con los exopoditos de las anténulas cambia de forma mudando al primer estado de phyllosoma. La liberación de las larvas ocurrió entre octubre y noviembre de cada año y en todos los casos después del atardecer (20:00 hr) y antes de la medianoche (23:30 hr). La hembra tiene una activa participación en la eclosión, siendo ella la que la induce. Extendiendo y levantando su abdomen en ángulo de $20^{\circ}$ respecto a la horizontal, agita sus pleópodos rítmicamente y genera una corriente que produce la expulsión de las larvas hacia atrás en ese mismo ángulo. Las larvas que son fuertemente fototácticas se dirigen a la superficie o hacia el lugar donde haya mayor luminosidad dentro de la penumbra. La hembra realiza este movimiento entre 3 a 5 veces en intervalos de 30 minutos durante la noche. Posteriormente cesa el movimiento hasta el siguiente día. Esta inducción de eclosión la realiza durante 3 a 5 días consecutivos, sin embargo el $90 \%$ de las larvas liberadas se produce antes de los tres días.

Aun cuando la eclosión ocurre durante las primeras horas de la noche, es posible obtener naupliosomas durante el día, extrayendo la hembra del estanque y aplicando un suave chorro de agua sobre sus pleópodos durante 5 a $10 \mathrm{seg}$. Esta maniobra se puede realizar 2 ó 3 veces por día.

Después de cinco días de iniciada la eclosión, se observó que entre un 2 y $8 \%$ de la masa ovífera permanece adherida a los pleópodos, además de gran cantidad de cápsulas vacías dejados por las larvas liberadas. La totalidad de estos huevos que permanecen adheridos se encuentran en el primer estado de desarrollo embrionario.

\section{Desarrollo larval}

Sólo se obtuvieron los seis primeros estados del desarrollo, los cuales demoraron entre 56 y 92 días dependiendo de la temperatura del cultivo (Fig. 6). Los cultivos realizados a $20^{\circ} \mathrm{C}$ demoraron 56 días en alcanzar el estado VI, con una regularidad de 1011 días entre cada uno de los estados; en cambio en el rango de $14-15^{\circ} \mathrm{C}$ y $19-20^{\circ} \mathrm{C}$ el tiempo que permanecen en cada estado varía entre 8 y 16 días y 




Figura 2. Huevo recientemente desovado de Jasus frontalis. Se observa el corion externo muy adhesivo separado inicialmente de la superficie del huevo (flechas). El otro extremo del corion se adhiere a la seda del pleópodo y comienza a alargarse $(*) .120 x$.

Figure 2. Early spawning egg of Jasus frontalis. It observe the sticky chorion (*) separated from the egg surface (arrows) at the beginning. The other side the chorion attached to the setae of pleopod (*). 120x.

Figura 3. Huevos adheridos a la seda del pleópodo después de $5 \mathrm{~min}$ post-desove. Se observa el corion adherido a la seta (*) pero aún no forma funiculus. 50x.

Figure 3. Attached egg to the setae of the pleopod $5 \mathrm{~min}$ after spawming. It is observed the chorion (*) attached to the setae without forming a funiculus. 50x.

Figura 4. Huevos adheridos a una seda (s) del pleópodo formando funiculus (f), después de 30 min postdesove. 50x.

Figure 4. Egg attached to setae (s) of the pleopod forming a funiculus after 30 min of spawning. 50x. 
entre 3 y 14 días, respectivamente. En todos los rangos de temperatura se observó que los dos primeros estados tienen una mayor duración que los estados intermedio (III y IV) y menor duración que los estados V y VI. La duración del phyllosoma I tiende a ser similar en temperaturas que van de 17 a $20^{\circ} \mathrm{C}$ (12 a 19 días). En cambio en el rango $14-15^{\circ} \mathrm{C}$ la duración fue de 38 días. Después del phyllosoma II, la duración de los estados varía de acuerdo a la temperatura del cultivo en una relación inversa. A mayor temperatura, menor tiempo de desarrollo (Fig. $6)$.

A pesar que los recambios de agua se realizaron diariamente y con agua filtrada a $10 \mu \mathrm{m}$, a partir del estado phyllosoma II se observó la presencia de hongos filamentosos adheridos a los pereiópodos y piezas bucales y especialmente a las sedas de los exopoditos nadadores de los pereiópodos (Fig. 7).

\section{DISCUSIÓN}

La muda de los machos ocurre entre febrero y marzo (Fig. 4), lo cual coincide con el comienzo de la disminución de la temperatura del agua (17,8 a $16,8^{\circ} \mathrm{C}$ ), en cambio, las hembras comienzan a mudar desde fines de abril, cuando la temperatura disminuye a $14,1^{\circ} \mathrm{C}$, hasta mediados de junio cuando la temperatura baja a $12,9^{\circ} \mathrm{C}$, es decir, al final de la disminución de temperatura, ya que en julio comienza a aumentar.
Según Arana y Martínez (1985), los mayores porcentajes de individuos blandos (machos y hembras) se encuentran en diciembre-enero y septiembre, siendo notoriamente más relevante la cantidad de machos en post-muda en diciembre-enero. Estos resultados coinciden con los resultados obtenidos en el presente estudio en los machos, aun cuando la muda está desfasada en un mes (febrero-marzo), ya que el proceso de muda comienza con el ablandamiento de las placas branqueostegales, proceso que va en aumento a medida que se acerca el momento de la ecdisis (Elorza y Dupré, 1996) y puede durar hasta 35 días. En las hembras, Arana et al. (1985) señalan que la mayor incidencia de ejemplares mudados se encuentra en mayo y junio, lo cual coincide plenamente con los presentes resultados.

Producida la muda, se determinó un crecimiento en longitud cefalotorácica de 1,3 a 3,6 mm, estableciéndose que las hembras aumentan de talla en mayor porcentaje $(2,7 \%)$ que los machos $(2,3 \%)$. Estos valores son inferiores a los calculados por Arana y Martínez (1985) mediante análisis de distribuciones de frecuencias de longitud cefalotorácica en esta misma especie, autores que señalan un incremento de $8,9 \mathrm{~mm}$ constante para todas las mudas, lo que equivale a una tasa porcentual decreciente en cada muda progresiva. También Backus (1960), al realizar el mismo análisis de frecuencia de distribución, estableció en Panulirus interruptus que el aumento de la longitud cefalotorácica en cada muda fue de 6,2 y 5,3 mm en machos y hembras respecti-

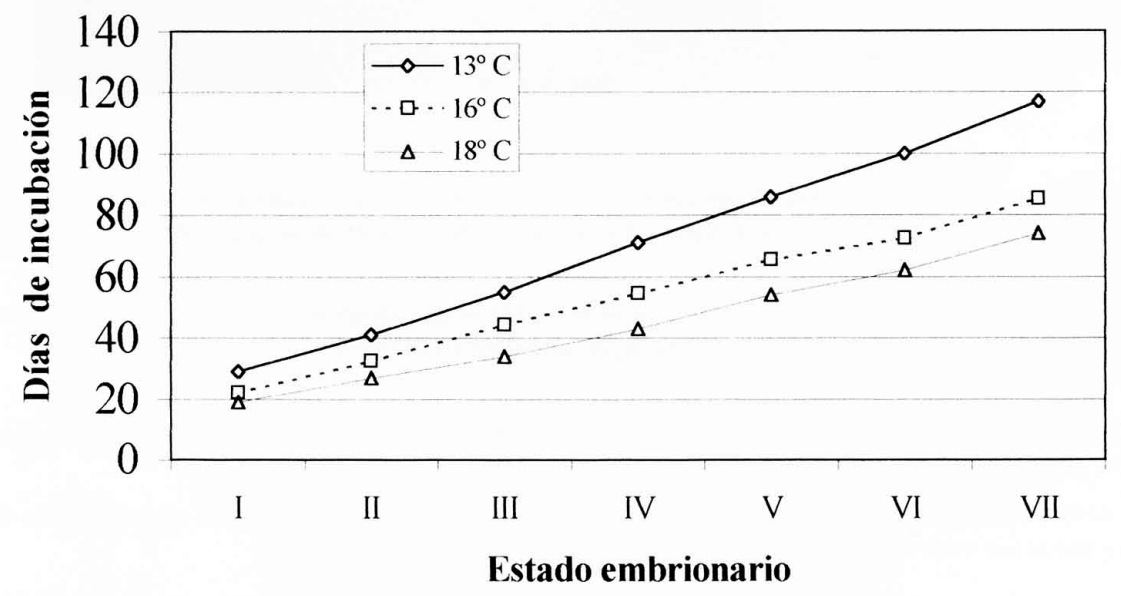

Figura 5. Desarrollo embrionario total a diferentes temperaturas de incubación.

Figure 5. Total embryonic development at different temperatures. 
vamente. El valor obtenido en esta oportunidad se asemeja más a los incrementos señalados en Jasus lalandii (Beyers, 1979).

Después de producida la ecdisis, el caparazón tiene una contextura blanda. En J. frontalis, esta contextura blanda al tacto se comienza a perder desde el primer día post-ecdisis hasta 3 a 5 días después, cuando se presenta rígido nuevamente. Este resultado difiere considerablemente de los señalado, para Jasus lalandii por Matthews (1962) y por Heydorn (1969) quienes respectivamente estimaron que el endurecimiento del caparazón demora 7-10 días y 15-32 días. Esta diferencia se podría deber a que los ejemplares en estudio fueron alimentados diariamente con alimentos que contenían altos niveles de $\mathrm{Ca}^{++}$.

Aun cuando no se pudo establecer el momento de apareamiento respecto al momento en que ocurrió la muda, se determinó que el desove de los huevos se produce después de un período de 21 días y que la cópula ocurrió entre individuos de tallas diferentes, siendo los machos de mayor talla que las hembras. La observación de que un solo macho sexualmente maduro pueda fecundar al menos a 6 hembras en un período de 2,5 meses, es de gran relevancia para futuros cultivos de esta especie.

El período en que las hembras portan huevos en los estanques comienza en junio y termina en octubre, lo cual coincide parcialmente con Arana et al. (1985) quienes señalan que en la isla Robinson Crusoe las hembras portan huevos entre julio y fe- brero del año siguiente, con la máxima incidencia entre octubre y diciembre. El adelantamiento del período de portación de huevos de los individuos en cautiverio (presente estudio) se debería a que la muda se produce anticipadamente, ya que el máximo desarrollo ovárico se alcanza en el período inmediatamente anterior a la ecdisis, debido a la intervención de la hormona vitelogenina que está íntimamente ligada al ciclo de muda. Este adelantamiento respecto a lo que ocurre en el medio natural (Arana et al., 1985) se debería a la rápida variación de temperatura que sufren los individuos en cautiverio. En cambio aquellos que se encuentran en el medio natural, entre los 100 y $200 \mathrm{~m}$ de profundidad donde la temperatura del agua presenta poca variación y la disminución o aumento ocurre más lento que en los estanques de cultivo, ya que éstos están sujetos a la variación de la temperatura de aguas superficiales con que éstos son alimentados.

La participación de la hembra en la eclosión de las larvas, mediante la agitación de sus pleópodos, sugiere que las larvas no son capaces de romper por sí mismas el corion que las envuelve, aun cuando este pierde rigidez por acción enzimática producida por las larvas hacia el final del desarrollo. La liberación ocurre por acción mecánica de los pleópodos de la hembra ya que no existe conexión directa entre el embrión y los pleópodos de la hembra (Tavonatti, 1998).

El conocimiento de la fecundidad de la especie permite estimar su potencial reproductivo, por cuan-

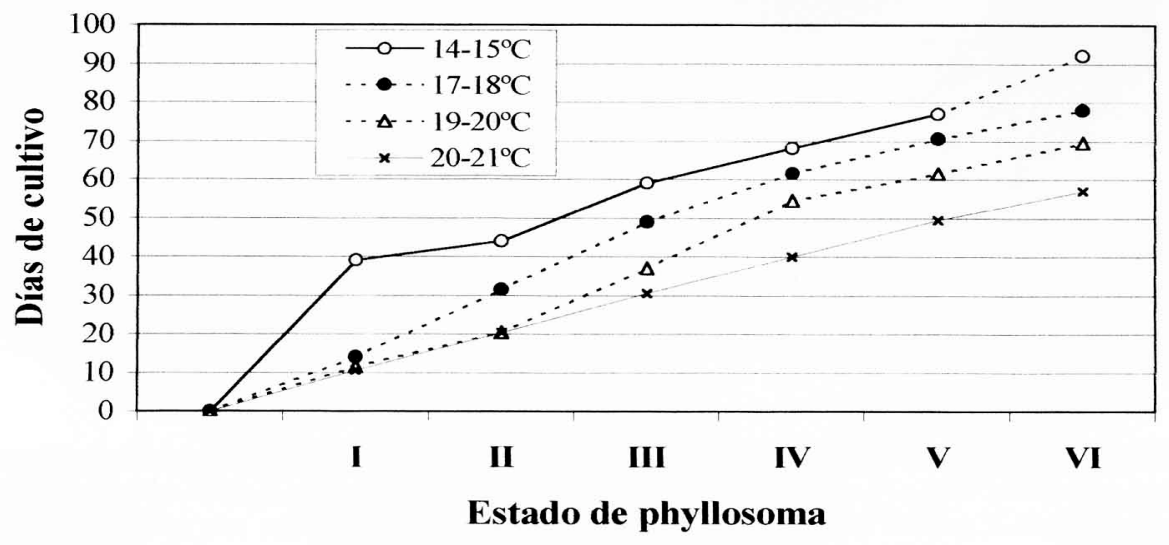

Figura 6. Estados de desarrollo larval de Jasus frontalis a diferentes temperaturas.

Figure 6. Larval development of Jasus frontalis at different temperatures. 


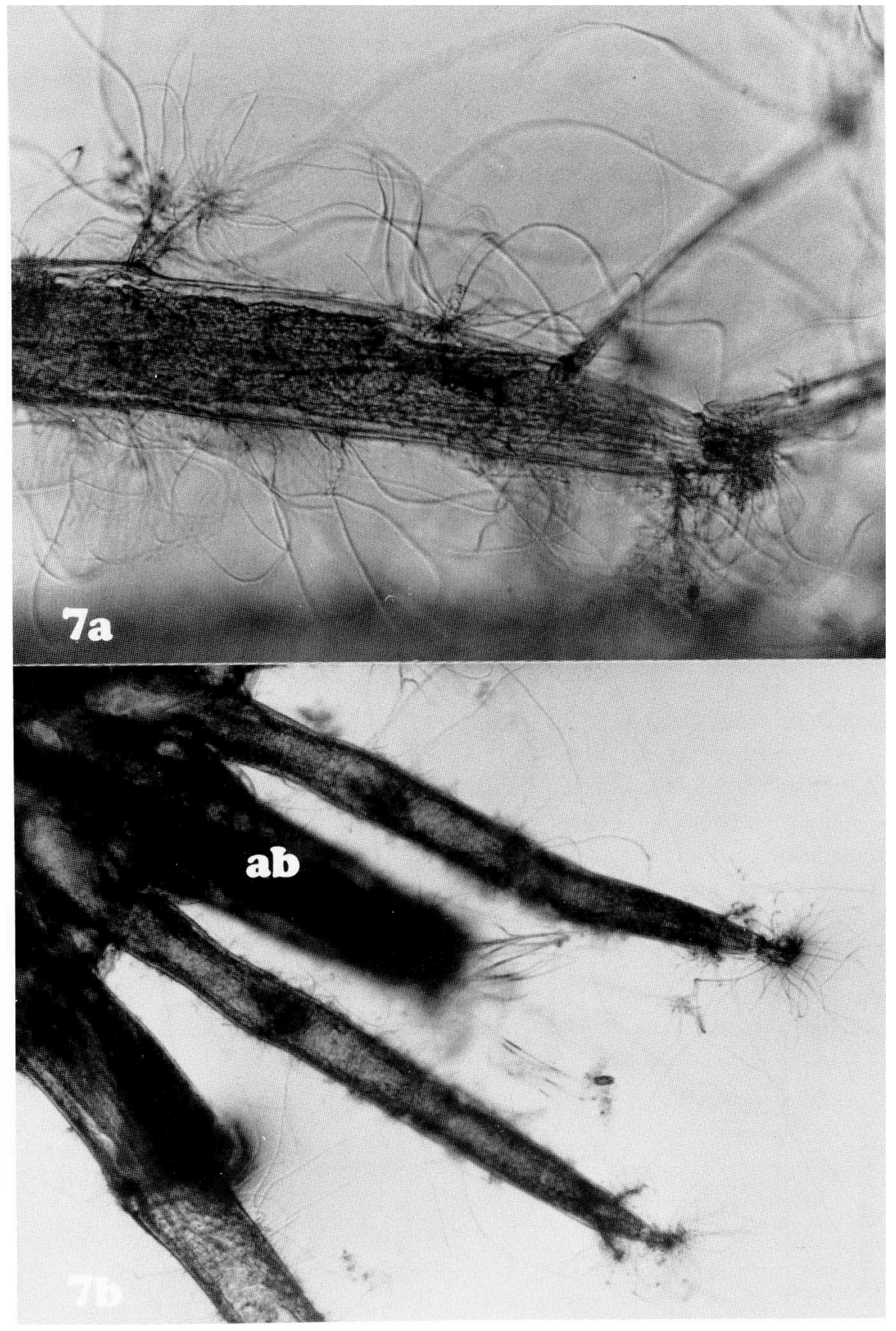

Figura 7. Phyllosoma en estado IV de Jasus frontalis. 7a: Extremo distal de la anténula con hongos filamentosos adheridos a su superficie. 240x. 7b: Extremo distal de la larva. Se observa el abdomen rudimentario de la larva (ab) y a ambos lados el cuarto par de pereiópodos en formación cubiertos son gran cantidad de hongos filamentosos. 100x.

Figure 7. Stage IV phyllosoma of Jasus frontalis. 7a: Distal end of the antennulae with filamentous fungi attached to the surface. 240x. 7b: Distal end of the larvae. It observe the rudimentary abdomen (ab) and the developing fourth pereiopod pair with attached filamentous fungi. 100x. 
to la cantidad de huevos representa, adecuadamente ponderada, el número de larvas que se puede integrar al plancton; sin embargo, en el presente estudio se determinó que entre un $2 \%$ y $8 \%$ de los huevos de masa ovífera permanece en el estado inicial del desarrollo (en estado I), lo que significa que la fecundidad real de la hembra es inferior a la calculada por la mayoría de los autores. Esto significa que el aporte real de larvas que hace a la población una hembra de 90-94 mm de longitud cefalotorácica, que porta entre 131.000 y 301.000 huevos (Arana et al., 1985) se verá disminuida entre 2.620-6.020 (2\%) y 10.480-24.080 (8\%) larvas respecto a las calculadas, cifra que debe sumarse a la pérdida de huevos que ocurre durante todo el desarrollo. En Paleomon pandaliformis y Macrobrachium acanthurus la pérdida de huevos alcanza al 23\% (Anger y Moreira, 1998).

La duración del desarrollo larval depende de la temperatura del cultivo. Se estableció una relación inversa entre el tiempo y la temperatura; no obstante, la mortalidad no fue la misma en todos los rangos de temperatura. Se observó una mejor sobrevivencia en larvas cultivadas entre $19-20^{\circ} \mathrm{C}$, esto debiéndose principalmente al aumento de la frecuencia de muda a mayor temperatura. Al mudar más rápido, se impide que haya una aumento en la formación de hongos filamentosos entre las sedas de los exopoditos de los pereiópodos y en el resto del cuerpo, permitiéndole desplazarse con mayor libertad y eficiencia para alimentarse.

Los resultados obtenidos permiten sugerir que el ciclo reproductivo en cautiverio es similar al registrado en el medio natural. Sin embargo, es necesario continuar con las investigaciones en este campo a fin de completar la información aportada en este estudio.

\section{REFERENCIAS}

Anger, K. y G. Moreira. 1998. Morphometric and reproductive traits of tropical caridean shrimps. J. Crust. Biol., 18: 823-838.

Arana, P., E. Dupré, y V. Gaete. 1982. Estructura poblacional, reproducción, talla de primera madurez sexual y fecundidad de la langosta Jasus frontalis. Estud. Doc., Univ. Católica Valparaíso, 2/82: $126 \mathrm{pp}$.

Arana, P., E. Dupré y V. Gaete. 1985. Ciclo reproductivo, talla de primera madurez sexual y fecundidad de la langosta Jasus frontalis. En: P. Arana (ed.). Investigaciones Marinas en el Archipiélago de Juan Fernández. Esc. Ciencias del Mar, UCV, Valparaíso, pp. 187-223.

Arana, P. y G. Martínez. 1985. Crecimiento por muda de la langosta de Juan Fernández (Jasus frontalis). En: P. Arana (ed.). Investigaciones Marinas en el Archipiélago de Juan Fernández. Esc. Ciencias del Mar, UCV, Valparaíso, pp. 225-236.

Arana, P. y C. Melo. 1973. La langosta de Juan Fernández. II. Pesca comercial de Jasus frontalis en la isla Santa Clara (1971-1972). Invest. Mar., 4: 135-154.

Arana, P. y C. Olate. 2000. Composición de las capturas de la langosta de Juan Fernández (Jasus frontalis) y determinación de parámetros biológico-pesqueros durante la temporada de pesca 19961997. Invest. Mar., Valparaíso, 28: 83-115.

Arana, P. y R. Vega. 2000. Esfuerzo, captura y captura por unidad de esfuerzo en la pesquería de la langosta de Juan Fernández (Jasus frontalis), durante la temporada de pesca 1996-1997. Invest. Mar., Valparaíso, 28: 117-133.

Backus, J. 1960. Observation on the growth rate of the spiny lobster. Calif. Fish Game, 46: 177-181.

Báez, P. 1973. Larvas phyllosomas del Pacífico sur oriental (Crustacea, Macrura, Scyllaridea). Rev. Biol. Mar., Valparaíso, 15: 115-130.

Beyers, C.J. 1979. Stock assessment and some morphometric and biological characteristics of the rock lobster Jasus lalandii on Marshall Rocks, its main commercial fishings area off South West Africa. 1971-1974. Investl. Rep. Sea Fish. Brch. S. Afr., 117: 1-26.

Díaz, P. y P. Arana. 1985. Estimaciones de mortalidades y de la edad crítica en la langosta de Juan Fernández (Jasus frontalis) de las islas Robinson Crusoe y Santa Clara. En: P. Arana (ed.). Investigaciones Marinas en el Archipiélago de Juan Fernández. Esc. Ciencias del Mar, UCV, Valparaíso, pp. 237-250.

Dupré, E. 1988. Desarrollo embrionario de la langosta de Juan Fernández Jasus frontalis (Decapoda, Macrura, Palinuridae). Invest. Mar., Valparaíso, 16: 49-62. 
Dupré, E. 1996. Primer estado de phyllosoma de la langosta de Juan Fernández, Jasus frontalis. Rev. Chil. Hist. Nat., 69: 231-242.

Dupré, E. y C. Guisado. 1996. Identificación de los primeros estados de phyllosoma de la langosta de Juan Fernández, Jasus frontalis. Invest. Mar., Valparaíso, 24: 39-50.

Elorza, A. 1998. Efecto de la 17-alfa-dihidroxiprogesterona sobre la maduración ovárica de la langosta de Juan Fernández, Jasus frontalis (Milne Edwards, 1837), (Crustacea, Decapoda; Palinuridae). Tesis para optar al Título de Biólogo Marino, Univ. Católica del Norte, 175 pp.

Elorza, A. y E. Dupré. 1996. Determinación de los estados del ciclo de muda en la langosta de Juan Fernández (Jasus frontalis Milne Edwards, 1837). Invest. Mar., Valparaíso, 24: 67-76.

Elorza, A. y E. Dupré. 2000. Arquitectura del ovario de la langosta de Juan Fernández, Jasus frontalis. Invest. Mar., Valparaíso, 28: 175-194.

Fisher, W.S., E.H. Nilson, L. Follett y R.A. Shleser. 1976. Hatching and rearing lobster larvae (Homarus americanus) in desease situation. Aquaculture, 7: 75-80.

Heydorn, A. 1969. The rock lobster of the South African west coast, Jasus lalandii (H. Milne Edwards). Notes on the reproductive biology and the determination of minimum size limits for commercial catches.. Dept. Commerce and Ind., Div. Sea Fisheries South Africa, Investl. Rep., 53: $1-32$.

Larraín, F. y E. Yañez. 1985. Diseño de un sistema de recolección y procesamiento de datos de captura y esfuerzo para la pesquería de langosta de Juan Fernandez (Jasus frontalis H. Milne Edwards, 1837). En: P. Arana (ed.). Investigaciones Marinas en el Archipiélago de Juan Fernández. Esc. Ciencias del Mar, UCV, Valparaíso, pp. 273-278.
Matthews, J.P. 1962. The rock lobster of South West Africa (Jasus lalandii) (Milne Edwards). Size frecuency, reproduction, distribution and availability. Administration of South West Africa Marine Research Lab., Investl. Rep., 7: 1-61.

Newman, G. y D. Pollock. 1974. Growth of rock lobster Jasus lalandii and its relationship to benthos. Mar. Biol., 24: 339-346.

Palma, S. 1985. Plancton marino de las aguas circundantes al archipiélago de Juan Fernández. En: P. Arana (ed.). Investigaciones Marinas en el Archipiélago de Juan Fernández. Esc. Ciencias del Mar, UCV, pp. 59-69.

Palma, S., J. Meruane y A. Mujica. 1976. Observaciones sobre el meroplacton del archipiélago de Juan Fernández. Enero 1974. Cienc. Tec. Mar, CONA 2: 117-126.

Tavonatti, S. 1998. Análisis del desarrollo embrionario de la langosta de Juan Fernández, Jasus frontalis, mediante microscopía electrónica. Tesis para optar al grado de Biólogo Marino, Univ. Católica del Norte, 143 pp.

Yañez, E., C. Canales y C. Silva. 2000. Evaluación indirecta y estado de explotación de la langosta (Jasus frontalis) en el archipiélago de Juan

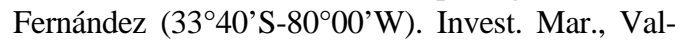
paraíso, 28: 135-148.

Yañez, E., L. Pizarro. M.A. Barbieri y O. Barra. 1985. Dinámica del stock de langosta (Jasus frontalis $\mathrm{H}$. Milne Edwards, 1837) explotado en el archipiélago de Juan Fernández (3340'S - 80 W). En: P. Arana (ed.). Investigaciones Marinas en el Archipiélago de Juan Fernández. Esc. Ciencias del Mar, UCV, Valparaíso, pp. 251-271. 